\title{
LAS DIMENSIONES HISTÓRICA Y LEGAL DE LA EAD EN BRASIL
}

Carla Maria Nogueira de Carvalho*

*Licenciada em Pedagogia e Estudos Sociais, Especialista em Metodologia do Ensino, Mestre em Educação pela Universidade Vale do Rio Verde ( UNINCOR) e Doutoranda em Ciência da Educação pela Universidade Nacional de Rosário ( UNR).Docente na UNINCOR - e-mail:profcarlamaria@oi.com.Br

\section{Recebido em: 30/10/2013 - Aprovado em: 15/12/2013 - Disponibilizado em: 15/01/2014}

RESUMEN: El presente trabajo describe el movimiento de ideas y acciones vividos en la educación a distancia en Brasil, buscando establecer conexión entre las dimensiones histórica y legal. La primera, mostrando las diversas experiencias construidas y realizadas a lo largo del tiempo y, la segunda, en la forma de articulación con la primera en cuanto condición real de implementación. La reflexión está basada en datos de pesquisa bibliográfica realizada en diferentes momentos de la historia de Brasil, desde la década del veinte hasta los días de hoy. El énfasis es dado en la forma de apropiación y de provecho de las propuestas de educación a distancia, así como en lo que puede ser construido, reinventándose la educación y la propia historia en favor de todos los brasileños.

Palabras-clave: Educación a distancia, historia, legislación y implementación.

RESUMO: O presente trabalho descreve o movimento de ideias e ações vivenciados na educação a distância no Brasil, buscando estabelecer conexão entre as dimensões histórica e legal. A primeira ,mostrando as diversas experiências construídas e realizadas ao longo do tempo e, a segunda, na forma de articulação com a primeira enquanto condição real de implementação. A reflexão está baseada em dados de pesquisa bibliográfica realizada em diferentes momentos da história do Brasil, desde a década de vinte até os dias de hoje. A ênfase é dada na forma de apropriação e de aproveitamento das propostas de educação a distância,bem como no que pode ser construído, reinventando-se a educação e a própria história a favor de todos os brasileiros.

Palavras-chave: Educação a distância, história, legislação e implementação.

\section{INTRODUCCIÓN}

"En la jerarquía de los problemas de una nación, nada sobrepasa en importancia, al de la educación." Ese lema del Manifiesto de los Educadores de 1932 inspira y sostiene la necesaria reflexión sobre los caminos y dimensiones recorridos por la nación brasileña en el movimiento de ideas y acciones vividos en su educación y, en el presente trabajo, el de la educación a distancia, una vez que la misma presenta posibilidades pedagógicas y sociales de extrema relevancia para un país continental y diverso como Brasil.

\section{DESARROLLO}

La iniciativa de intercambiar informaciones, vehicular orientaciones e instrucciones entre personas espacialmente distantes es constatada desde la antigüedad. En Grecia y, posteriormente, en Roma personas se comunicaban a través de correspondencia (correo) para intercambio y/o transmisión de informaciones útiles tanto para el cotidiano privado como para el desarrollo económico, político y social de las comunidades, así como para informaciones científicas. Ese epistolario greco-romano va a 
manifestarse en el Cristianismo naciente y, atravesando los siglos, adquiere especial desarrollo en los períodos del Humanismo y del Iluminismo. Sin embargo, la impartición de saberes específicos sin la relación presencial entre profesor y alumno sucede en la modernidad. El perfeccionamiento de los servicios de correo, la agilización de los medios de transporte y, sobretodo, el desarrollo tecnológico aplicado al campo de la comunicación y de la información influyeron decisivamente en los destinos de la educación a distancia.

Saraiva (1996) indica el anuncio de un curso de taquigrafía publicado en el periódico de Boston, en los Estados Unidos, en 1728, como uno de los primeros marcos de la EAD. Hace referencia a cursos por correspondencia en Suecia (1833) e Inglaterra (1840) y apunta en Berlín (1856), una acción institucionalizada que crea la primera escuela de lenguas por correspondencia.

Al final del siglo XIX hubo un crecimiento significativo de implantación de instituciones de EAD, donde la Universidad Abierta del Reino Unido (Open University) se destacó al resaltar la importancia de la EAD como enfrentamiento de la presión social para mayor acceso a la enseñanza superior.
En Brasil, su evolución histórica es marcada por el surgimiento y diseminación de los medios de comunicación. En la exposición conmemorativa del Centenario de la Independencia, en 1922, la radio telefonía es mostrada al país. Edgard Roquette-Pinto $^{1}$ se entusiasmó con los nuevos equipos y, al comienzo de 1923, convicto de la importancia educativo cultural de la radio, creó, en 20 de abril, la Radio Sociedad de Rio de Janeiro para promover la educación por radiodifusión, marcando así el inicio de la EAD en Brasil.

En 1934, también Roquette-Pinto, fundó la Radio Escuela Municipal de Rio de Janeiro, emisora de carácter estrictamente educacional actuando en los varios niveles de enseñanza. Sin embargo, sólo a partir de 1960 son encontrados registros de programas de EAD sistematizados por el Gobierno Federal, como el contracto entre el MEC (Ministerio de Educación y Cultura) y la CNBB (Conferencia Nacional de los Obispos de Brasil) para la expansión del sistema de escuelas radiofónicas a los estados nordestinos, surgiendo de ahí el Movimiento de Educación de Base (MEB) como sistema de enseñanza a distancia no

\footnotetext{
${ }^{1}$ Médico, antropólogo y educador brasileño. Fue el precursor de la radiodifusión brasileña, siempre con el objetivo de difundir cultura y educación.
} 
formal y el establecido en la propia estructura del MEC, el Programa Nacional de Teleducación.

Aquí cabe recordar que la primera Ley de Directrices y Bases de la Educación Nacional $(\mathrm{LDB})^{2}$ fue creada en 1961, seguida por una versión en 1971. La primera, publicada en 20 de diciembre de 1961, por el Presidente João Goulart, casi treinta años después de ser prevista por la Constitución de 1934, permitiendo, en su artículo 104, la enseñanza experimental. La segunda, publicada en 11 de agosto de 1971, durante el régimen militar, por el Presidente Emílio Garrastazu Médici, apuntando, en su artículo 25, la educación a distancia como posible modalidad de la enseñanza supletoria.

Los años de 1964 a 1985 corresponden a una fase de autoritarismo y represión del Brasil República, se trata del Estado Autoritario-Militar implantado por el Golpe Militar de 31 de marzo de 1964.

Sin embargo, coherente con Pimentel (1995), hay hechos relevantes relacionados con la EAD, tales como : el empiezo de los trabajos de la Comisión para Estudios y Planeamiento de la Radiodifusión Educativa, en 1965; la instalación de ocho emisoras de

\footnotetext{
${ }^{2}$ LDB, define y regulariza el sistema de educación brasileño con base en los principios presentes en la Constitución.
}

televisión educativa (TV Universitaria de Pernambuco, TV Educativa de Rio de Janeiro, TV Cultura de São Paulo, TV Educativa de Amazonas, TV Educativa de Maranhão , TV Universitaria del Rio Grande del Norte, TV Educativa de Espírito Santo y TV Educativa del Rio Grande del Sul), de 1966 a 1974; la creación de la Fundación Padre Anchieta, mantenida por el Estado de São Paulo, con actividades educativas y culturales a través de la radio y de la televisión y de la Fundación Educacional Padre Landell de Moura (FEPLAN), institución privada sin fines lucrativos, que promovió la educación de adultos a través de teleducación por multimedios, en 1967; el Instituto de radiodifusión del estado de Bahía para la educación básica y secundaria y para la formación de profesores, así como el sistema de Televisión Educativa (TVE) de Maranhão/CEMA (Centro Educativo de Maranhão), ambos en 1969, siendo que este último hasta hoy ofrece, en recepción organizada, con el apoyo de orientadores de aprendizaje, estudios de $5^{\circ}$ a $8^{\circ}$ años de la enseñanza fundamental, utilizando programas de televisión y material impreso, que permiten profundizar los contenidos trabajados y realizar pesquisas; la obligatoriedad, para las emisoras 
comerciales de radio y televisión, de la transmisión gratuita de cinco programas semanales de treinta minutos diarios, de lunes a viernes, o con setenta y cinco minutos los sábados y domingos, a través de la Portaría 408 de 1970. También en este año es iniciada, en cadena nacional, la serie de cursos del Proyecto Minerva, irradiando los cursos de Capacitación Fundamental y Madurez Fundamental, producidos por la FEPLAN y Fundación Padre Anchieta; nace la Asociación Brasileña de Teleducación (ABT), en 1971, pionera en cursos a distancia, capacitando los profesores a través de correspondencia; fue creado, en 1972, incluso, en la estructura del Ministerio de la Educación y Cultura, el Programa Nacional de Teleducación (Prontel), a quien competía coordinar y apoyar la teleducación en Brasil; Proyecto Minerva pasa a producir, en 1973, el Curso Supletorio de $1^{\circ}$ Grado; Proyecto Saci, conclusión de los estudios para el Curso Supletorio "João da Silva", en 1973-74, bajo formato de telenovela, para la enseñanza de los cuatro primeros años del $1^{\circ}$ grado. El curso introdujo una innovación pionera en el mundo, un proyecto piloto de tele didáctica de la TVE que logró el premio especial del Jurado Internacional del Premio Japón. Otro producto importante derivado del
Saci fue la implementación de un curso de maestreado en Tecnología Educacional; TVE Ceará empieza , en 1974, a generar tele clases y el Centro de Enseñanza Técnica de Brasilia (CETEB) inicia el planeamiento de cursos en convenio con la Petrobras para la capacitación de los empleados de esta empresa y del Proyecto Logus II, en convenio con el MEC, para habilitar profesores desconocedores sin aislarlos del ejercicio docente; lanzado el Tele curso de $2^{\circ}$ Grado, en 1978, por la Fundación Padre Anchieta (TV Cultura-São Paulo) y Fundación Roberto Marinho, con programas televisivos, apoyados por fascículos impresos, para preparar al tele alumno para los exámenes supletorio; creación de la Fundación Centro Brasileiro de Televisión Educativa/MEC (FCBTVE), en 1979, dando continuidad al Curso "João da Silva", surge aún el Proyecto Conquista, también como telenovela, para los últimos años del primer grado y empieza la utilización de los programas de alfabetización por TV, denominado MOBRAL, en recepción organizada, controlada o libre, abarcando todas las capitales de los estados de Brasil; es implantado, en carácter experimental, de 1979 a 1983, el Posgrado (Pos Graduación Tutorial a Distancia) por la CAPES (Coordinación de 
Perfeccionamiento del Personal de Enseñanza Superior del MEC), administrado por la Asociación Brasileña de Tecnología Educacional (ABT), con el objetivo de capacitar docentes universitarios del interior del país; la FCBTVE cambió su sigla, en 1981, para FUNTEVE coordinando las actividades de la TV Educativa de Rio de Janeiro, de la Radio MEC-Rio, de la Radio MEC-Brasilia, del Centro de Cine Educativo y del Centro de Informática Educativa; creación de la TV Educativa del Mato Grosso del Sul y empiezo del Proyecto Ipé, de la Secretaria de la Educación del Estado de São Paulo y de la Fundación Padre Anchieta, con cursos para actualización y perfeccionamiento del magisterio de $1^{\mathrm{o}}$ e $2^{\mathrm{o}}$ Grados, utilizándose de multimedios, en 1983/1984.

Sobretodo a partir de las décadas de 60 y 70, la tele educación, aunque manteniendo los materiales escritos como base, pasa a incorporar, el audio y el videocasete, las transmisiones de radio y televisión, el videotexto, el videodisco, el ordenador $\mathrm{y}$, recientemente, la tecnología de multimedios, que combina textos, sonidos, imágenes, mecanismos de generación de caminos alternativos de aprendizaje (hipertextos, diferentes lenguajes), instrumentos de una fijación de aprendizaje con feedback inmediato, programas tutoriales informatizados, entre otros.

A finales de la década de 80 Brasil vive hechos históricos importantes, entre ellos la reunión, a partir de 1987, de la Asamblea Nacional Constituyente que preparó la nueva Constitución, promulgada en 1988. La misma estableció en los artículos 205 a 214 nuevas directrices para la educación. A partir de ahí la LDB anterior fue considerada obsoleta y se inició una serie de debates para la elaboración de la nueva ley.

En este período son acciones significativas en la historia de la EAD: el curso por correspondencia "Verso y Reverso-Educando el Educador", creado en 1988 por el MEC/Fundación Nacional para Educación de Jóvenes y Adultos (EDUCAR),para capacitación de profesores da educación básica de jóvenes y adultos, con apoyo de programas televisivos a través de la Red Manchete; el Proyecto Ipé pasa a enfatizar los contenidos curriculares, en 1991 y, en este mismo año, la Fundación Roquete Pinto, la Secretaría Nacional de Educación Básica y Secretarias Estatales de Educación implantan el Programa de Actualización de Docentes, abarcando los cuatro primeros años de la enseñanza 
fundamental y alumnos de los cursos de formación de profesores. En la segunda fase, el proyecto fue denominado "Un salto para el futuro".

De acuerdo con Saraiva (2005) ese proyecto, "Un salto para el futuro", representa un marco importante en la historia de la EAD y de la televisión educativa brasileña, por el alcance nacional de la utilización, por la concepción y formato del programa, que permite la interactividad, por la acción integrada y coordinada de varios órganos, además de constituirse un instrumento eficaz para lo alcanzable de una de las metas de la política educacional - la educación continuada de los profesores de la enseñanza fundamental, con vistas a su permanente actualización, a la mejora de la productividad del sistema escolar y a la garantía de la calidad de la educación.

Además de esto, "Un salto para el futuro", por su importancia, alcance y resultados, fue estimulador de cambio de mentalidad y de desarrollo de acciones concretas, que abrieron nuevas perspectivas para la EAD en el país.

En el primer caso, propició a miles de profesores la intimidad con el proceso educativo vencedor de distancias y con diversidad de soluciones de uso pedagógico de las tecnologías de comunicación. Y fue a partir de su alcance nacional y de los resultados alcanzados que se abrieron nuevas perspectivas para la EAD en Brasil.

En 1992, el Núcleo de Educación a Distancia del Instituto de Educación de la Universidad Federal de Mato Grosso (UFMG) en conjunto con la Universidad Estadual de Mato Grosso y la Secretaria de Estado de Educación y con apoyo de la Tele-Université $d u$ Quebec (Canadá) crea el proyecto de Licenciatura Plena en Educación Básica: $1^{\circ}$ a $4^{\circ}$ años del $1^{\circ}$ grado, utilizando la EAD. El curso fue iniciado en 1995.

El gobierno brasileño, a través del MEC y del Ministerio de las Comunicaciones (MC), tomó, a partir de 1993, las primeras medidas concretas para la formulación de una política nacional de EAD, para la creación, a través del Decreto ${ }^{\circ} 1.237$, de 6/9/94, del Sistema Nacional de Educación a Distancia, además de muchas otras: Protocolo de Cooperación $n^{\circ} 3 / 93$, firmado entre el MEC y el MC, con la participación del Consejo de Rectores de las Universidades Brasileiras (Crub), del Consejo de Secretarios de Educación (Consed) y de la Unión Nacional de Dirigentes Municipales de Educación (Undime), visando el desarrollo de un sistema nacional de 
EAD; Convenio $n^{\circ} \quad 6 / 93 \quad-$ MEC/MC/Embratel, con la participación del Ministerio de la Ciencia y Tecnología (MCT), del Ministerio de la Industria y Comercio (MinC), Crub, Consed y Undime, para garantizar la viabilidad del EAD; Acuerdo de Cooperación Técnica 4/93 MEC/UnB, para la coordinación, por la UnB, de un Consorcio Interuniversitario, con la finalidad de dar soporte científico y técnico a la educación básica, utilizando los recursos de la Educación Continuada y de la Educación a Distancia; Decreto creando la Televía para la Educación, firmado por el presidente de la República y ministros de la Educación y de las Comunicaciones, con deducción del valor de tarifas para programas de EAD; Creación de la Coordinación Nacional de EAD, en el ámbito del MEC.

Además de esas medidas, a nivel federal, "Un salto para el futuro" propició a la creación, en cada unidad federada, de una Coordinación de Educación a Distancia vinculada a la respectiva Secretaría Estatal de Educación, encargada de la utilización de programas de EAD.

En 1995, el hito fue el lanzamiento de la TV Escuela, programa concebido y coordinado por el MEC, en ámbito nacional. Su objetivo es el perfeccionamiento y la valoración de los profesores de la red pública y la mejora de la calidad de la enseñanza, por medio de una cadena de televisión dedicado exclusivamente a la educación; fue creada, en 1996, en la estructura del MEC, la Secretaria de Educación a Distancia, para dar soporte a la creciente investida de las instituciones educacionales rumbo a la EAD.

El 20 de diciembre de 1996, la nueva LDB, Ley n9394, elaborada por los Senadores Darcy Ribeiro y Mauricio Correa en articulación con el poder ejecutivo a través del MEC, fue sancionada por el presidente Fernando Henrique Cardoso y por el ministro de la educación Paulo Renato. En ella fueron establecidas las bases legales para la EAD en Brasil, constando en el artículo 80 que: el Poder Público incentivará el desarrollo y la conducción de programas de enseñanza a distancia, en todos los niveles $\mathrm{y}$ modalidades de enseñanza y de educación continuada; y en el título VIII, de las Disposiciones Generales que: la educación a distancia será ofrecida por instituciones específicamente acreditadas por la Unión; y que cabrá a la Unión reglamentar requisito para realización de exámenes, para registro de 
diplomas relativos a cursos de educación a distancia.

A partir de la nueva LDB las propuestas educativas comprometidas con la superación del tiempo y del espacio, que se llamaron enseñanza por correspondencia y tele educación, pasan a asumir la responsabilidad de ser educación y de realizarse a distancia. En ese sentido la EAD pasó a ser considerada alternativa regular $-\mathrm{y}$ reglamentada - de prestación educacional a los brasileños. Sale del grupo de proyectos experimentales y pasa a ser estrategia de acceso a la educación de calidad y a la democratización de enseñanza, derecho del ciudadano y deber del Estado y de la Sociedad, asegurados por la legislación.

Vale recordar que de la tensión entre tradición y modernización, la educación virtual abierta y en red viene, gradualmente, avanzando como forma especializada y diferenciada de educación cuya especificidad le confiere una dinámica de formación profesional mundial. Formación esa que puede resignificar y/o desarrollar nuevas competencias técnicas y humanas a favor de las necesidades actuales de la sociedad a nivel local y global.

En Brasil, visando ampliar el número de plazas en la educación superior, promover la formación inicial y continuada a los profesionales del magisterio y a los profesionales de la administración pública, ha sido creado e implementado, por el Ministerio de la Educación, junto con los Estados, Municipios y Universidades Públicas de Enseñanza Superior, el Sistema Universidad Abierta de Brasil (UAB) para ofrecer cursos de Graduación, Posgrado y de Extensión Universitaria.

Este Sistema fue concretizado por el Ministerio de Educación en el año de 2005, en acuerdo con la ANDIFES (Asociación Nacional de los Dirigentes de las Instituciones Federales de Enseñanza Superior) y Empresas Estatales, en el ámbito del Foro de las Estatales por la Educación con foco en las Políticas y en la Gestión de la Educación Superior. Se trata de una política pública de formación de profesores con articulación entre la Secretaría de Educación a Distancia SEED/MEC y la Directoría de Educación a Distancia - DED/CAPES (Coordinación de Perfeccionamiento de Personal de Nivel Superior) con vistas a la expansión de la educación superior, en el ámbito del Plan de Desarrollo de Educación - PDE.

Instituido vía decreto ministerial $\mathrm{N}^{\circ}$.5.800 de 8 de junio de 2006, el Sistema UAB tiene, desde su génesis, la 
intención de contribuir para la democratización de la educación.

Hoy, la UAB, agrega 92 Instituciones de Enseñanza Superior ofreciendo casi 450 cursos para aproximadamente 220.000 estudiantes, 557 polos de apoyo presencial y la Directoria de Educación a Distancia de la CAPES, o sea, no es una universidad sino un sistema que congrega universidades.

Este sistema abre las puertas de las universidades federales para una población que muchas veces no tendría condiciones de ir hasta el local, lo que podría y puede cambiar la vida de las personas y del país. Así, los cursos del Sistema son ofrecidos en la modalidad a distancia, semi presenciales, y buscan sostenerse en el referencial teórico denominado constructivo colaborativo.

En la educación abierta o universidad abierta, la introducción de las tecnologías de la información y de la comunicación ha originado una proliferación de experiencias que imponen la necesidad de un conocimiento mayor de sus características, evolución y perspectivas de desarrollo con la intención de contribuir para la mejora de la calidad en la educación virtual.

\section{CONCLUSIÓN}

Desde la década de 20, Brasil viene construyendo su historia de EAD. Vivimos la transmisión radiofónica, la enseñanza por correspondencia, la televisiva; utilizamos la informática hasta los actuales procesos de utilización conjugada de medios - la telemática y la multimedia. Estamos asistiendo, al inicio del $3^{\circ}$ milenio, el consenso de que un país con la dimensión y las características del nuestro tiene que romper las amarras del sistema convencional de enseñanza y buscar formas alternativas para garantizar que la educación inicial y continuada sea realmente derecho de todos.

En este contexto, la EAD despunta como posibilidad, probablemente viviendo nuevas etapas, con énfasis en la integración de medios, buscando la mejor y mayor interactividad. Pero sea cual sea la tecnología adoptada, la EAD tendrá que tener, siempre, una finalidad educativa, concibiéndose aquí, en conformidad con Freire, el acto educativo como posibilidad, como un hacer que está siendo, sostenido en el inédito viable y en la propia no conclusión del ser humano, siempre en potencia, vuelto a la vocación para el ser más. 
Se apunta, tras ese breve registro, que lo esencial en la historia de la EAD en Brasil no es lo que ya fue realizado, sino lo que puede ser realizado en la

\section{REFERENCIAS BIBLIOGRÁFICAS}

GOMES,C.A.Darcy

Ribeiro.Recife:Massangana,2010.

GOMEZ,M.V.Re-leitura de Paulo Freire para uma teoría da informática naeducação.Contato,Brasília,n.3,jun.19 99.

Cibercultura, formación $y$ actuación docente en red: guía para profesores. Brasilia: Liberlivro,2010.

LEVY, P. Educación y Cybercultura.1998. Recuperado el 23 de abril de 2011,de www. caosmose.net/pierrelevy leducaecyber.html.

PIMENTEL.EADnoBrasil.1995.Recupe rado el 14 de noviembre de 2011, de www.webartigos.com/artigos/educacaoa-distancia.

RAMA,C.La Educación Superior Virtual en América Latina y el Caribe.

2004.Recuperado el 2 de marzo de 2011, de www.iesalc.unesco.org.ve.

SARAIVA,T. A Educação a Distância no Brasil. Brasília:Em Aberto, 1996.

SARAIVA,V.Educação Cultura.2005.Recuperado el 20 de diciembre de 2011, de www.tvebrasil.com.br/salto. relación dialéctica con el otro y con el mundo, reinventándose la educación y la propia historia en favor de todos los brasileños. 